\title{
Measurement of effective thermal conductivity of compacted granular media by the transient plane source technique
}

\author{
Weijing Dai ${ }^{1}$, Yixiang Gan ${ }^{1, *}$ \\ ${ }^{1}$ School of Civil Engineering, the University of Sydney, Darlington, Sydney, Australia
}

\begin{abstract}
To successfully realise industrial applications handling granular media, especially those involving heating and cooling processes, the temperature fields must be properly evaluated according to the accurate thermal properties of the media. The knowledge the effective thermal conductivity is regarded as one of the fundamental aspects. However, due to the complicated relations between the effective thermal conductivity and the heterogeneity and complexity in the structures and composition of the granular media, the quantitative prediction of the conductivity is challenging. Therefore, experimental investigation of the effective thermal conductivity becomes desired and this can provide first-hand data for industrial reference and serve as the benchmark for the theoretical analysis. In this study, the transient plane source technique is employed to investigate the effective thermal conductivity of compacted granular beds by the application of the commercially available Hot Disk system. The granular beds of different particle size ranges are characterised under different mechanical loading conditions by different sensors. Experimental results are discussed and suggestion to achieve reliable experimental designs is provided.
\end{abstract}

\section{Introduction}

Granular media, for a long time, is one of the most important material systems widely used in industry including chemical synthesis, food production, thermal insulation, additive manufacturing and other application consisting of granular beds. Efforts have been devoted to investigate the properties and features of granular media to provide knowledge for the realisation and optimisation of those applications. One step further into them, it is clear that the thermal transport is a crucial physical phenomenon determining the capability of not only the high-end technologies but also the conventional industries. For example, powder beds based additive manufacturing, promising in fabricating products of complex geometries, requires precise control of the thermal energy input into the processed beds to selectively melt a target region [1]; thermal management techniques of lithium battery systems is critical due to the safety concern in the application of high power and fast charging [2]. A growing demand on the appropriate knowledge about the effective thermal conductivity of granular media rises because the effective thermal conductivity of the media is one of the key properties to evaluate the thermal transport phenomenon [3]. With knowing the effective thermal conductivity of beds, numerical simulations can be effectively employed to investigate the potential issues in real scenarios without costly actual implementation [4,5]. Besides, the micromechanisms that are difficult to detect in-situ can be validated by investigating the correlation between the mechanisms and the accessible effective thermal conductivity [6]. Considerable progresses have been made in the field of the characterisation methods for the effective thermal conductivity of granular media and catalysed the development of the theories to analyse the measured data.

Theoretically, the Zehner-Schlunder-Bauer model used a unit cell constructed by two particle halves and filling fluid phase to represent a granular media according to the packing properties of the media. The effective thermal conductivity of granular media is derived by the parallelisation rule of the thermal resistance of the thermal transport components of the unit cell $[7,8]$. This model has been later modified [9] and adopted [6] widely by other researchers to study related problems. Besides the evolution of theories, experimental research about a variety of granular media is of equal importance. Unsaturated sand and soil have been measured by the steady-state probe method [10,11], but the attributes of those granular media is hard to interpret, limiting the understanding of the thermal transport mechanisms involved. Hot wire method was employed to measure the effective thermal conductivity of glass beds at different ambient pressured [12] and it is observed the effective thermal conductivity increased with the increasing ambient pressure. The effect of applied mechanical loading onto granular beds has been studied by Tehranian, et al [13] and Widenfeld, et al [14], applying the steady-state measurement techniques. Recently, the transient plane source technique has been developed to measure the effective thermal conductivity 
of particulate materials. Anisotropic effect on the effect thermal conductivity of granular beds introduced by the uniaxial compression was observed by Mo et al [15]. Using a similar technique, Liang et al [16] found a sensitive dependency of the effective thermal conductivity of sands on the amount of hydrates. Since this transient plane source technique attracts more attention in the community working with granular media, some questions that may be encountered in the practise of this technique remain unclear and have not been discussed previously by the researchers. In general, this technique comes with different sized sensors. The size contrast between the sensor and the particles has potential influence on the measurement. In this study glass beads of relative low thermal conductivity and steel ball bearings of relative high conductivity are utilised to investigate the dependency of the effective thermal conductivity upon the particle size. The response of effective thermal conductivity of these granular materials subjected to mechanical loading is tested. Finally, we will address the influence of the sensor size and comment on the selection of the sensor according to our measurement.

\section{Experimental setup}

\subsection{Transient plane source technique}

The transient plane source technique utilises a thin metal stripe or a metal disk to act as a continuous plane heat source and at the same time to serve as a resistive temperature sensor [17]. In principle, the temperature profile during the heating period of the sensor is sensitive to the surrounding materials. Therefore, by monitoring the resistance of the sensor which reflects the temperature change over the heating period, the thermal properties of the materials surrounding the metal film can be extracted. The advantages of this transient measurement technique over steady-state measurement technique are: (1) short measurement time, (2) little restriction of the geometries of samples, (3) non-invasive requirement on the sample structure, (4) no dependency on the measured temperature gradient $\nabla T$. Based on this transient technique, the hot disk sensor was introduced [18] to measure thermal conductivity, thermal diffusivity and specific heat of different kinds of materials. This sensor adopts a double spiral nickel ring for spontaneously sensing and heating. This nickel ring is sealed inside an insulated film to prevent the electrical leakage and the potential damage to the metal. After that, the Hot Disk systems integrating the sensor with the electrical measurement equipment gains popularity in the thermal properties measurement. Various kinds of materials, like solid, liquid, gel [19], and powder beds [15], have been measured by the Hot Disk systems.

\subsection{Measurement kit for granular beds}

A measurement kit is designed and fabricated to facilitate the experiments on granular beds. As illustrated in Figure 1, two stainless steel rings of $2 \mathrm{~cm}$ height form a cylindrical sample holder with an inner diameter of 37 $\mathrm{cm}$. The sensor is placed between two rings with the sensor plane parallel to the cross-section of the rings. The granular materials are poured into the lower ring first, and then the surface of the particle bed is flattened to ensure the horizontal placement of the flexible sensor. The other ring is stacked onto the top of lower ring. The inner space of the ring is filled with the particles and the top surface is flattened as well. The mass of the filling granules is recorded. With the density of the bulk material known, the packing factor of the granular beds can be obtained by calculating the ratio between the solid volume of particles and the total volume of the inner space of two rings. A short rod with diameter slightly being smaller than the inner diameter of rings can be used to apply the external mechanical loading onto the top surface of the granular bed. Currently, the increase of the mechanical loading is realised by stacking weights onto the rod.

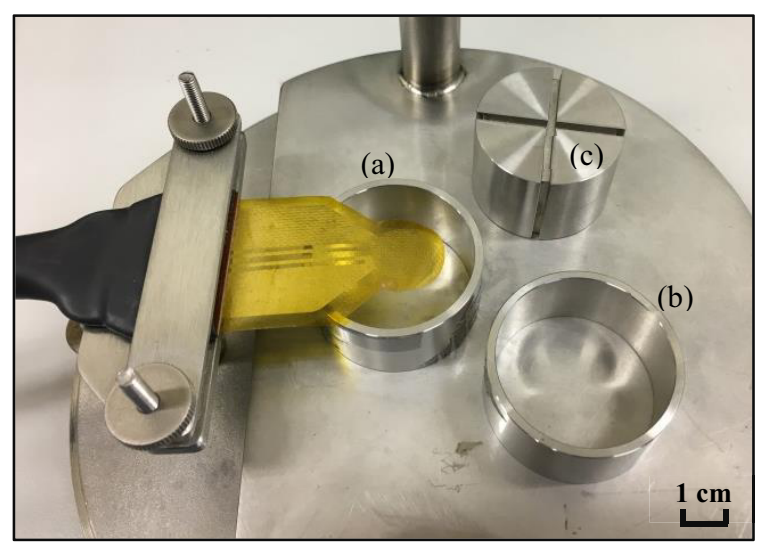

Figure 1. The simple measurement kit including two rings (a) and (b) and a short steel rod to support weights (c).

\subsection{Types of granular beds}

In this study, six glass beds of different diameters, e.g.. (1) $0.1 \sim 0.2 \mathrm{~mm}$, (2) $0.25 \sim 0.5 \mathrm{~mm}$, (3) $0.5 \sim 0.75 \mathrm{~mm}$, (4) $0.75 \sim 1.0 \mathrm{~mm}$, (5) $1.0 \sim 1.25 \mathrm{~mm}$ and 6) $1.25 \sim 1.5 \mathrm{~mm}$, are measured by using the same testing procedures. Optimal procedures are selected for the following study to produce reliable measurement. Hot disk sensors of two sizes are used to test the potential variations caused by the sensor size. The change in the effective thermal conductivity of glass beds subjected to external mechanical loadings is investigated by stacking different weights. Moreover, granular beds of different sized (1 $\mathrm{mm}$ and $2 \mathrm{~mm}$ ) stainless steel balls are measured following the similar procedures.

\section{Experimental result and discussion}

The assembly of the measurement kit is shown in Figure 2 with half of the cylindrical sample holder filled by the glass beads of diameters ranging from $0.75 \mathrm{~mm}$ to 1.00 $\mathrm{mm}$. Two sensors \#5465 and \#5501 of different sizes are shown in the Figure 2 (a) and (b) respectively, and the size contrast can be seen by the comparison between the sensor and the rings. 


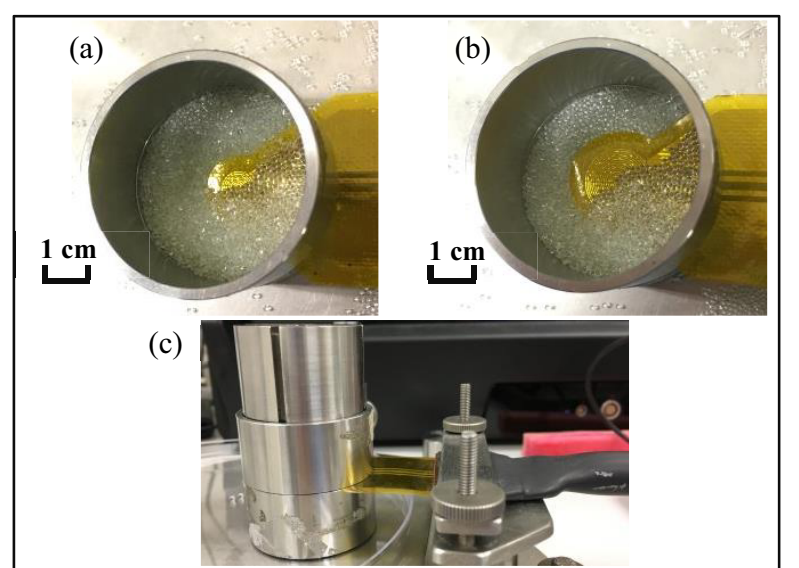

Figure 2. The assemblies of the measurement kit. (a) \#5465 sensor, the diameter is $3.189 \mathrm{~mm}$; (b) \#5501 sensor, the diameter is $6.403 \mathrm{~mm}$; (c) the assembly for applying mechanical loading.

The effective thermal conductivity with respect to the granule size is measured first. It can be seen from Figure 3 that the effective thermal conductivity increases with the increasing of the granule size. This increasing trend is consistent with the result shown by our numerical study [20] and this phenomenon can be explained by the Smoluschowski effect which is originally observed as the decrease of the effective thermal conductivity of the porous media during evacuation of gases from the media. Generally, this effect is introduced by the increase of the Knudsen number, the ratio of the mean free path of gas molecules over the dimension of a space filled by the gas. The studies of the variable ambient pressure proved the effective thermal conductivity of granular materials is reduced by the increase of the mean free path of the gas molecules [12,21]. In our study, although the gas pressure of the beds is the nearly constant air atmosphere pressure, the Smoluschowski effect is induced by the decrease of the pore space due to the reduction of the granule size. This decrease increases the Knudsen number, as is achieved by the decrease of the gas pressure in other studies. One thing shown by the measurement result is worth to notice that the small sensor tends to give a larger effective thermal conductivity. This indicates that the measured value may depend on the size of the sensor used.

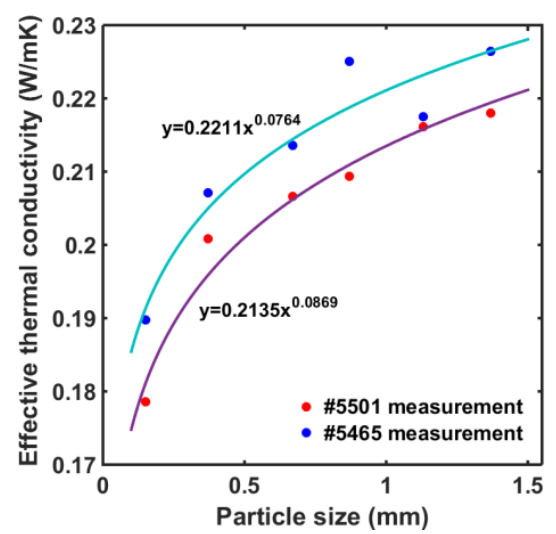

Figure 3. The effective thermal conductivity of glass beds consisting of beads in different diameter ranges. The horizontal coordinates of the points corresponding to the mid-point of the size range.
The effective thermal conductivity versus packing factor of the beds in this study is plotted in Figure 4. The material properties of the glass beds were input into the Zehner-Schlunder-Bauer model to calculate the effective thermal conductivity. The calculated conductivity versus packing factor is also plotted in the Figure 4. A higher packing factor generally means a higher effective thermal conductivity. The measured result deviates from the model prediction when the packing factor over 0.6. Because the Zehner-Schlunder-Bauer model does not take the Smoluschowski effect into account, it may not be able to fully capture the scenario of this study, in particular for these beds with smaller granules. However, as the packing factor has not been well controlled in current step of this study, the scattering of effective thermal conductivity in the packing factor range $0.60 \sim 0.63$ require further work to interpret.

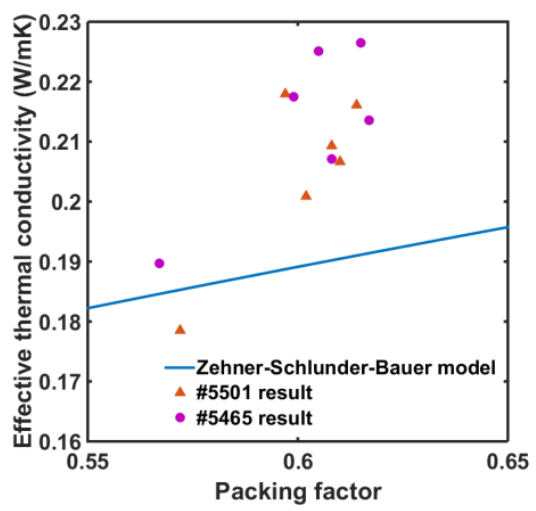

Figure 4. The measured effective thermal conductivity of beds versus packing factors. The solid line shows the calculated Zehner-Schlunder-Bauer conductivity of the same beds.

Then three kinds of glass beds (small--0.1 0.2 mm, medium--0.5 0.75 mm, large--1.25 1.5 mm) were selected to investigate the influence of the mechanical loading. The effective thermal conductivity of glass beds shows a slight increment with increasing the mechanical loading in the tested range. This increase is relative obvious when the loading was just increased from 0 to about $20 \mathrm{kPa}$. Further increase shows a minor effect. Again the granule size effect can be observed.

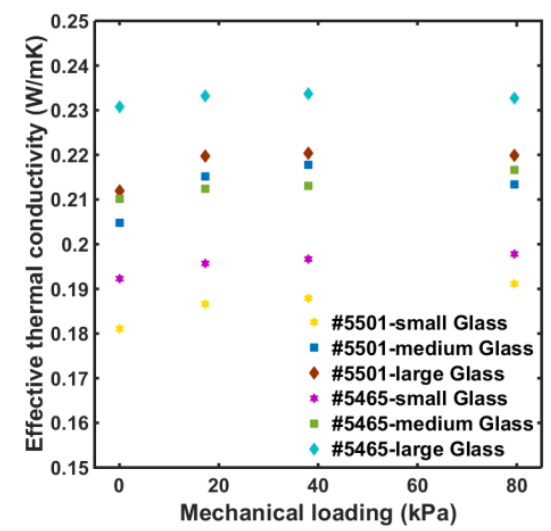

Figure 5 . The effective thermal conductivity versus the external mechanical loadings for glass beds.

In parallel, the steel beds were studies in the same way as glass beds. In contrast to the result from the glass beds, the enhancement in the effective thermal 
conductivity occurs in the entire mechanical loading range. This continuous rise in the conductivity can be resulted from the relatively high thermal conductivity of steel compared to the glass. The higher loading pressure is supposed to enlarge the contact area between particles, giving rise to the conduction through the solid phase. Similar to the glass beds, a larger particle size shows a higher effective thermal conductivity.

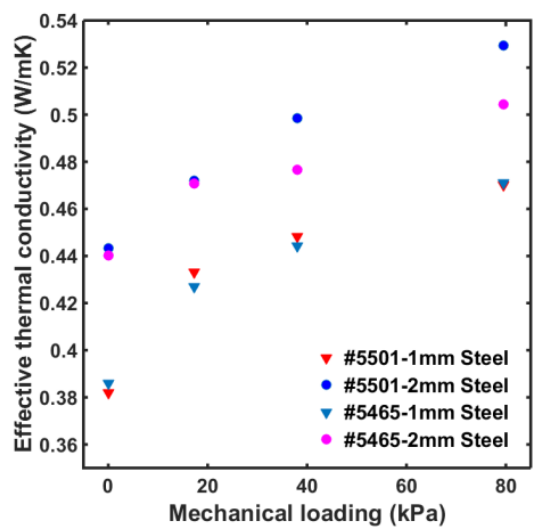

Figure 6 . The effective thermal conductivity versus the external mechanical loadings for steel beds.

In terms of the influence of the sensor size, it is obvious in the glass beds measurement, but looks like playing no significant role in the steel beds measurement. It may be possible that the low thermal conductivity of the glass, producing a localised heating profile, is responsible for this observation. However, conclusive comments are hard to given in this stage. Further experimental study combined with numerical simulation method is required to clarify this influence so that the measured results can be better understood.

\section{Conclusion}

The effective thermal conductivity of glass beds as well as steel beds has been measured by the Hot Disk system based on the transient plane source technique. It is clearly demonstrated that the effective thermal conductivity of the granular beds is influenced by the granule size and it increases with the increasing granule size. This phenomenon can be explained by the Smoluschowski effect on the thermal conductivity of interstitial gases. The lowest thermal conductivity of $0.1 \sim 0.2 \mathrm{~mm}$ glass beds can also be attributed to the lowest packing factor. It is worth to study how the packing factor varies the effective thermal conductivity in a more controllable experiment procedure. The mechanical loading plays a positive role in enhancing the thermal transport process in the measured beds. However, such a positive effect is more significant in the steel beds compared to the glass beds because the intrinsic thermal conductivity of steel is much higher than the glass.

The variation in the measured effective thermal conductivity by using sensors of different sizes is observed in this study, in particular for beds with intrinsic materials having lower conductivity, subjected to the measurement conductivity by the Hot Disk system. Further research is needed to provide a sound theoretical understanding and fundamental explanation to this phenomenon.

Acknowledgements: Financial support for this research from the Australia Research Council through grant DE130101639 is greatly appreciated.

\section{References}

1 M. Markl and C. Körner, Annual Review of Materials Research 46 (1), 93 (2016).

K. Zhao, M. Pharr, J. J. Vlassak, and Z. Suo, Journal of Applied Physics 108 (7), 073517 (2010).

Y. Gan and M. Kamlah, Journal of the Mechanics and Physics of Solids 58 (2), 129 (2010).

4 A. Bauereiß, T. Scharowsky, and C. Körner, Journal of Materials Processing Technology 214 (11), 2522 (2014).

5 Y. Gan, Francisco Hernandez, Dorian Hanaor, Ratna Annabattula, Marc Kamlah and Pavel Pereslavtsev, Fusion Science \& Technology 66 (1), 83 (2014).

6 M. Bahrami, M. M. Yovanovich, and J. R. Culham, International Journal of Heat and Mass Transfer 49 (19-20), 3691 (2006).

$7 \quad$ P. Zehner and S. EU, Chemie Ingenieur Technik 42 (14), 933 (1970).

8 R. Bauer and E. Schlunder, International Chemical Engineering 18 (2), 189 (1978).

9 C. T. Hsu, P. Cheng, and I. W. Wong, International Journal of Heat and Mass Transfer 37 (17), 2751 (1994).

10 S. Yagi and D. Kunii, AIChE Journal 3 (3), 373 (1957).

11 W. Woodside and J. H. Messmer, Journal of Applied Physics 32 (9), 1688 (1961).

12 M. A. Presley and P. R. Christensen, Journal of Geophysical Research: Planets 102 (E3), 6551 (1997).

13 Fatollah Tehranian and M. A. Abdou, Fusion Science \& Technology 27 (1995).

14 G. Widenfeld, Y. Weiss, and H. Kalman, Powder Technology 133 (1-3), 15 (2003).

15 J. Mo, D. Garrett, and H. Ban, International Journal of Thermophysics (2015).

16 D. Li and D. Liang, International Journal of Heat and Mass Transfer 92, 8 (2016).

17 S. E. Gustafsson, Review of Scientific Instruments 62 (3), 797 (1991).

18 M. Gustavsson, E. Karawacki, and S. E. Gustafsson, Review of Scientific Instruments 65 (12), 3856 (1994).

19 Y. He, Thermochimica Acta 436 (1-2), 122 (2005).

20 W. Dai, Y. Gan, and D. Hanaor, Submitted.

21 A. V. Gusarov and E. P. Kovalev, Physical Review B 80 (2), 024202(1) (2009). 